\title{
Association between Urinary Cadmium-to-Zinc Intake Ratio and Adult Mortality in a Follow-Up Study of NHANES 1988-1994 and 1999-2004
}

\author{
Kijoon Kim ${ }^{1,2}$, Melissa M. Melough ${ }^{1}$, Junichi R. Sakaki ${ }^{1}$, Kyungho Ha $^{1}{ }^{1}$, Dalia Marmash ${ }^{1}$, \\ Hwayoung Noh $^{3}\left(\mathbb{D}\right.$ and Ock K. Chun ${ }^{1, *(1)}$ \\ 1 Department of Nutritional Sciences, University of Connecticut, Storrs, CT 06269, USA; \\ drkijoon@gmail.com (K.K.); melissa.melough@uconn.edu (M.M.M.); junichi.sakaki@uconn.edu (J.R.S.); \\ kyungho.ha@uconn.edu (K.H.); dalia.marmash@uconn.edu (D.M.) \\ 2 Department of Food and Nutrition, Sookmyung Women's University, Seoul 04310, Korea \\ 3 Nutritional Methodology and Biostatistics Group, Section of Nutrition and Metabolism, \\ International Agency for Research on Cancer(IARC-WHO), 69372 Lyon, France; nohh@fellows.iarc.fr \\ * Correspondence: ock.chun@uconn.edu; Tel.: +1-860-486-6275
}

Received: 19 November 2019; Accepted: 19 December 2019; Published: 24 December 2019

\begin{abstract}
Cadmium (Cd) is a toxic heavy metal associated with increased mortality, but the effect of zinc $(\mathrm{Zn})$ intake on the association between $\mathrm{Cd}$ and mortality is unknown. The objective of this study was to examine the association of urinary $\mathrm{Cd}$ to $\mathrm{Zn}$ intake ratio $(\mathrm{Cd} / \mathrm{Zn}$ ratio) and mortality risk. In total, 15642 US adults in NHANES 1988-1994 and 1999-2004 were followed until 2011 (15-year mean follow-up). Of the 5367 total deaths, 1194 were attributed to cancer and 1677 were attributed to CVD. After adjustment for potential confounders, positive associations were observed between urinary $\mathrm{Cd}$ and all-cause mortality (HR for highest vs. lowest quartile: $1.38 ; 95 \% \mathrm{CI}$ : 1.14-1.68) and cancer mortality (HR: 1.54; CI: 1.05-2.27). Urinary Cd was positively associated with cancer mortality among the lowest $\mathrm{Zn}$ consumers, and the association diminished among the highest $\mathrm{Zn}$ consumers. Positive relationships were observed between the $\mathrm{Cd} / \mathrm{Zn}$ ratio and all-cause mortality (HR: 1.54; CI: 1.23-1.93), cancer mortality (HR: 1.65; CI: 1.11-2.47) and CVD mortality (HR: 1.49; CI: 1.18-1.88). In conclusion, these findings indicate that $\mathrm{Zn}$ intake may modify the association between $\mathrm{Cd}$ and mortality. Furthermore, the $\mathrm{Cd} / \mathrm{Zn}$ ratio, which was positively associated with mortality from all causes, cancer, and CVD, may be an important predictor of mortality.
\end{abstract}

Keywords: zinc intake; urinary cadmium; mortality; NHANES; urinary Cd/Zn intake ratio

\section{Introduction}

During certain industrial processes, cadmium (Cd), a highly toxic and carcinogenic metal, is produced as a byproduct. Cd can then leach into soil and water which are used in agricultural processes, causing $\mathrm{Cd}$ to appear in the food supply [1]. The food supply is the primary source of $\mathrm{Cd}$ exposure in non-smokers, and tobacco is an additional prime source of $\mathrm{Cd}$ in smokers [2]. Due to its long half-life, $\mathrm{Cd}$ may be accumulated in multiple tissue types in the human body, thereby leading to many diseases such as kidney dysfunction [3], reproductive dysfunction [4], cardiovascular disease [5], diabetes [6], osteoporosis [7], cancer [8], and increased mortality [9].

Higher Cd exposure has been linked to increased cardiovascular disease (CVD) risk in a Swedish cohort study [10] and linked to heart failure and mortality in elderly Australian women [11]. Cd exposure has also been associated with mortality from total, lung and pancreatic cancers in the Strong Heart Study, which included 4545 American Indian participants [12]. Several studies have reported that 
higher Cd exposure was associated with increased mortality from all causes, cancer, and CVD in the general populations of Japan [13], Belgium [14], and the US [15].

Interestingly, the mechanism underlying Cd's effect on these diseases relates to its interaction with zinc ( $\mathrm{Zn})$. Cd competes with $\mathrm{Zn}$ in cellular uptake and metallothionein and due to its higher affinity for metallothionein, $\mathrm{Cd}$ displaces $\mathrm{Zn}$, resulting in increased synthesis of metallothionein and $\mathrm{Cd}$ absorption [16]. Increased saturation of $\mathrm{Cd}$ at binding sites within the kidney can lead to microscopic tubular proteinuria (known to occur at urinary Cd concentrations of $2 \mu \mathrm{g} / \mathrm{g}$ creatinine) and renal dysfunction (at urinary Cd concentrations of $10 \mu \mathrm{g} / \mathrm{g}$ creatinine) [1,17]. Due to the competition between $\mathrm{Cd}$ and $\mathrm{Zn}$ for several key binding sites, dietary $\mathrm{Zn}$ may interfere in the absorption, accumulation, and bioavailability of $\mathrm{Cd}$ in the body. This has been demonstrated in several animal studies where Zn supplementation protected against Cd toxicity in the spleen, liver, and kidney [18-20]. Epidemiologic evidence indicates that Zn intake is linked to lower Cd burden in US adults, presumably due its competitive uptake and metallothionein binding with $\mathrm{Cd}$ [21]. Among US men with low $\mathrm{Zn}$ consumption and high concentrations of $\mathrm{Cd}$ excreted in urine, total prostate-specific antigen, a biomarker indicative of prostate cancer, was elevated, suggesting a relationship between dietary $\mathrm{Zn}$, urinary $\mathrm{Cd}$ and cancer [22]. Cancer mortality risk appears to be higher among those with inadequate Zn intake [23].

These studies suggest that dietary Zn may protect against $\mathrm{Cd}$ toxicity in humans, indicating that dietary $\mathrm{Zn}$ should be considered in investigating the association between $\mathrm{Cd}$ exposure and health outcomes, including mortality. There are few studies exploring the relationship between the Cd/Zn ratio and mortality from all causes, cancer, and CVD. Therefore, the objective of this study was to investigate the association of the $\mathrm{Cd} / \mathrm{Zn}$ ratio with mortality from all causes, cancer, and CVD among US adults.

\section{Materials and Methods}

\subsection{Study Population}

Data from 15,641 participants in NHANES 1988-1994 [24] and 1999-2004 [25-27] were used. Exclusion criteria consisted of pregnant or breastfeeding women $(n=458)$, those with unreliable or incomplete dietary recalls $(n=1128)$ and those with missing data on urinary creatinine and urinary $\mathrm{Cd}$ ( $n=7957)$. Additionally, because cancer and CVD incidence and mortality rates are very low for young people $[28,29]$, we excluded subjects under 30 years old. NHANES study protocols were approved by the National Center for Health Statistics research ethics review board. (Institutional Review Board approval and documented consent from participants for NHANES 1988-1994 and Protocol \#98-12 for NHANES 1999-2004).

\subsection{Estimation of Dietary Zn Intake}

Dietary Zn intake was estimated from one 24-h dietary recall in NHANES 1988-1994 and 1999-2004.

\subsection{Biomarker Data}

Participants' spot urine samples were collected in the mobile examination center. Urinary Cd was measured as described in the NHANES Laboratory Procedures Manual [30,31]. While elevated blood $\mathrm{Cd}$ confirms recent acute exposure, it does not correlate well with total body burden. Therefore, urinary $\mathrm{Cd}$, which reflects integrated exposure and total body burden [32], was used in this analysis. Urinary $\mathrm{Cd}$ concentrations were determined using inductively coupled plasma-mass spectrometry (ICP-MS) and adjusted by creatinine.

\subsection{Mortality Data Linked to the NHANES}

We combined NHANES data from the survey years 1988-1994 and 1999-2004 with their linked mortality datasets. During a mean of follow-up of 15 years (through 31 December 2011), 5367 deaths 
occurred [33]. NHANES-linked mortality data were ascertained through probabilistic record matching with the National Death Index (NDI). Follow-up time per person was calculated beginning at the time of the NHANES interview date and ending at death or censoring. The causes of death were determined from the 10th revision of the International Statistical Classification of Diseases and Related Health Problems [34]. The number of deaths, crude mortality rate and age-standardized mortality rate (ASMRs) of all-cause, cancer and CVD according to quartile of urinary Cd were estimated. ASMRs were based on US Census 2000 population data.

\subsection{Statistical Analysis}

All statistical analyses were performed using SAS software, version 9.4 (SAS Institute Inc., Cary, NC, USA). To account for the complex survey design, the appropriate SAS procedures, weight, strata, and cluster variables were used. Participants were grouped into quartiles based on urinary $\mathrm{Cd}$ concentration after adjustment for urinary creatinine and into tertiles based on dietary $\mathrm{Zn}$ intake for 1988-1994 and 1999-2004. The frequencies and means of sociodemographic and lifestyle factors according to quartiles of urinary $\mathrm{Cd}$ concentration were estimated. Significance testing for the differences in urinary $\mathrm{Cd}$ between subgroups was conducted by ANOVA or chi-square test.

Poverty income ratio (PIR) was calculated as a ratio of household income to the poverty threshold after accounting for inflation and family size, based on poverty guidelines provided by the Department of Health and Human Services (HHS). Participants were subsequently dichotomized as PIR $\leq 1.3$ or $>1.3$. Positive smoking status was defined as having at least 100 lifetime cigarettes. Current smokers were defined as those who did not quit smoking and were stratified as heavy ( $\geq 15$ cigarettes daily) and light ( $<15$ cigarettes daily). Former smokers were identified as those who had reported quitting smoking by the time of interview. Diabetes mellitus was defined as having a fasting plasma glucose level $>126 \mathrm{mg} / \mathrm{dl}$ or taking insulin or diabetic medications to lower blood sugar. Hypertension was defined as having a systolic blood pressure $>140 \mathrm{mmHg}$, diastolic pressure $>90 \mathrm{mmHg}$, or taking any prescribed medication for the treatment of high blood pressure. Aspirin users were defined as those using aspirin regularly during the past month. Supplement users were defined as those using nutritional supplements during the past month by the time of the baseline interview.

The interaction between $\mathrm{Zn}$ intake and urinary $\mathrm{Cd}$ was tested for significance in Cox proportional hazards model. Hazard ratios (HRs), multivariable HRs and 95\% confidence intervals (CIs) for all-cause, cancer and CVD mortality were estimated by quartile of urinary $\mathrm{Cd}$, quartile of urinary Cd stratified by level of dietary $\mathrm{Zn}$, and quartile of $\mathrm{Cd} / \mathrm{Zn}$ ratio. Multivariable model 1 was adjusted for age and gender. Model 2 additionally included ethnicity, body mass index (BMI), PIR, smoking status, diabetes, hypertension, alcohol consumption, saturated fatty acid intake, $\mathrm{Zn}$ intake, aspirin use, supplement use and history of CVD and cancer. Alcohol consumption was included as a covariate based on previous studies [35,36]. Covariates were selected for all cause-specific mortalities based on the existing literature. Model 3 of cancer mortality included all variables in Model 2 except hypertension, saturated fatty acid intake and history of CVD [37,38]. Multivariable model 4 of CVD mortality included all variables of Model 2 except Zn intake and history of cancer [39-41]. All $p$-values reported are two-sided $(\alpha=0.05)$.

\section{Results}

When separated into quartiles of Cd exposure using urinary $\mathrm{Cd}$, US adults in the highest quartile were more likely to be women, older, current heavy smokers, and aspirin users compared to those with lower $\mathrm{Cd}$ exposures. Those in the highest quartile of $\mathrm{Cd}$ exposure also had lower incomes, lower BMIs, and had lower rates of hypertension and histories of cancer, coronary heart disease (CHD) or stroke. Adults in the lowest quartile of urinary $\mathrm{Cd}$ were more likely to be men, of younger age, non-smokers, have higher levels of income, and higher intakes of $\mathrm{Zn}$ and saturated fat (Table 1). 
Table 1. Baseline characteristics of US adults $\geq 30 \mathrm{y}$ in NHANES 1988-1994 and 1999-2004 by quartile of urinary Cd concentration $(n=15,641)$.

\begin{tabular}{|c|c|c|c|c|c|c|c|c|c|c|}
\hline & \multicolumn{4}{|c|}{ NHANES 1988-1994 } & \multirow[b]{3}{*}{$p$-Value ** } & \multicolumn{4}{|c|}{ NHANES 1999-2004 } & \multirow{3}{*}{$p$-Value $* *$} \\
\hline & \multicolumn{4}{|c|}{ Urinary Cd Concentration ( $\mu \mathrm{g} / \mathrm{g}$ Creatinine) (Range) } & & \multicolumn{4}{|c|}{ Urinary Cd Concentration ( $\mu \mathrm{g} / \mathrm{g}$ Creatinine) (Range) } & \\
\hline & $\begin{array}{c}\mathrm{Q1}^{*} \\
(n=3033) \\
(0.00-0.29)\end{array}$ & $\begin{array}{c}\mathrm{Q}^{*} \\
(n=3034) \\
(0.29-0.52)\end{array}$ & $\begin{array}{c}\mathrm{Q} 3{ }^{*} \\
(n=3035) \\
(0.52-0.90)\end{array}$ & $\begin{array}{c}\mathrm{Q} 4^{*} \\
(n=3033) \\
(0.90-23.35)\end{array}$ & & $\begin{array}{c}\mathrm{Q1}^{*} \\
(n=876) \\
(0-0.22)\end{array}$ & $\begin{array}{c}\mathrm{Q} 2{ }^{*} \\
(n=877) \\
(0.22-0.37)\end{array}$ & $\begin{array}{c}\mathrm{Q} 3{ }^{*} \\
(n=877) \\
(0.37-0.62)\end{array}$ & $\begin{array}{c}\mathrm{Q} 4^{*} \\
(n=876) \\
(0.62-4.20)\end{array}$ & \\
\hline Follow-up time, mean, years & $19.0(0.2)$ & $17.9(0.2)$ & $16.9(0.3)$ & $15.4(0.2)$ & $<0.0001$ & $9.6(0.1)$ & $9.5(0.2)$ & $9.1(0.1)$ & $8.8(0.2)$ & $<0.0001$ \\
\hline Age, mean, years & $43.0(0.4)$ & $49.1(0.5)$ & $53.9(0.6)$ & $57.8(0.6)$ & $<0.0001$ & $44.7(0.6)$ & $51.3(0.6)$ & $54.8(0.7)$ & $57.8(0.7)$ & $<0.0001$ \\
\hline Men, \% & 57.2 & 49.8 & 43.5 & 35.8 & $<0.0001$ & 63.0 & 45.2 & 40.0 & 33.7 & $<0.0001$ \\
\hline Ethnicity & & & & & $<0.0001$ & & & & & 0.073 \\
\hline White, \% & 86.1 & 82.3 & 83.3 & 86.7 & & 81.5 & 83.0 & 84.9 & 83.1 & \\
\hline Black, \% & 9.0 & 13.0 & 12.3 & 9.9 & & 11.1 & 10.6 & 10.0 & 11.9 & \\
\hline Mexican-American, $\%$ & 4.9 & 4.7 & 4.4 & 3.4 & & 7.4 & 6.4 & 5.1 & 4.9 & \\
\hline BMI & & & & & $<0.0005$ & & & & & $<0.005$ \\
\hline $\mathrm{BMI}<25, \%$ & 40.9 & 37.5 & 38.1 & 44.2 & & 27.4 & 24.1 & 30.1 & 39.8 & \\
\hline $25 \leq \mathrm{BMI}<30, \%$ & 35.0 & 35.0 & 34.4 & 35.0 & & 39.1 & 39.9 & 38.2 & 34.0 & \\
\hline $\mathrm{BMI} \geq 30, \%$ & 24.1 & 27.5 & 27.5 & 20.8 & & 33.5 & 36.0 & 31.7 & 26.2 & \\
\hline Smoking ${ }^{1}$ & & & & & $<0.0001$ & & & & & $<0.0001$ \\
\hline Never & 63.2 & 48.3 & 34.5 & 22.5 & & 67.5 & 55.8 & 40.1 & 24.5 & \\
\hline Former & 25.4 & 32.2 & 32.9 & 32.7 & & 24.7 & 30.0 & 35.4 & 34.2 & \\
\hline Current $(<15$ cigarettes/d) & 6.1 & 7.5 & 8.4 & 9.3 & & 5.2 & 7.6 & 9.3 & 10.8 & \\
\hline Current $(\geq 15$ cigarettes/d) & 5.3 & 12.0 & 24.2 & 34.5 & & 2.6 & 6.6 & 15.2 & 30.5 & \\
\hline $\mathrm{PIR}^{2}>1.3, \%$ & 88.7 & 84.7 & 82.1 & 77.7 & $<0.0001$ & 86.7 & 83.2 & 79.2 & 74.4 & $<0.0005$ \\
\hline Diabetes mellitus ${ }^{3}, \%$ & 5.7 & 7.7 & 8.3 & 10.4 & $<0.0001$ & 14.9 & 14.1 & 20.0 & 15.7 & 0.374 \\
\hline Hypertension ${ }^{4}, \%$ & 22.2 & 33.7 & 40.6 & 47.6 & $<0.0001$ & 26.2 & 37.5 & 44.0 & 45.6 & $<0.0001$ \\
\hline Aspirin use ${ }^{5}, \%$ & 8.4 & 12.6 & 15.3 & 18.4 & $<0.0001$ & 10.5 & 13.8 & 15.6 & 21.6 & $<0.001$ \\
\hline Supplement use ${ }^{6}, \%$ & 46.6 & 43.1 & 42.2 & 41.1 & 0.0695 & 54.3 & 59.2 & 58.8 & 57.9 & 0.307 \\
\hline History of $\mathrm{CHD}^{7}$ or stroke, $\%$ & 1.7 & 3.5 & 5.8 & 8.2 & $<0.0001$ & 2.3 & 4.9 & 7.3 & 10.7 & $<0.0001$ \\
\hline History of cancer, $\%$ & 4.2 & 9.2 & 12.8 & 13.8 & $<0.0001$ & 7.6 & 8.3 & 11.6 & 14.4 & $<0.001$ \\
\hline \multicolumn{11}{|l|}{ Dietary intake, mean } \\
\hline Zinc, $\mathrm{mg} / \mathrm{d}$ & $12.7(0.2)$ & $11.8(0.2)$ & $11.6(0.4)$ & $10.7(0.4)$ & $<0.01$ & $13.5(0.5)$ & $11.7(0.3)$ & $11.1(0.4)$ & $10.7(0.5)$ & $<0.0005$ \\
\hline Alcohol, g/d & $10.4(1.0)$ & $8.7(0.8)$ & $10.0(1.2)$ & $9.5(0.9)$ & 0.112 & $12.6(1.4)$ & $10.8(1.7)$ & $10.1(1.8)$ & $10.6(1.7)$ & 0.311 \\
\hline Saturated fats, g/d & $29.5(0.5)$ & $27.0(0.5)$ & $25.6(0.8)$ & $24.5(0.4)$ & $<0.0001$ & $30.3(1.0)$ & $26.1(0.8)$ & $24.2(0.7)$ & $23.3(0.6)$ & $<0.0001$ \\
\hline
\end{tabular}

* First quartile (Q1), second quartile (Q2), third quartile (Q3) and fourth quartile (Q4) ${ }^{* *}$ Tested by ANOVA or chi-square test. ${ }^{1}$ Smokers defined as those who smoked at least 100 cigarettes in lifetime, former smokers defined as smokers who quit. ${ }^{2}$ Poverty income ratio. ${ }^{3}$ Diabetes defined as fasting plasma glucose level greater than $126 \mathrm{mg} / \mathrm{dl}$ or taking medication including insulin and/or medication to lower blood sugar. ${ }^{4}$ Hypertension was defined as systolic blood pressure exceeding $140 \mathrm{mmHg}$ or diastolic pressure over $90 \mathrm{mmHg}$ or taking prescribed medicine for high blood pressure. ${ }^{5}$ Aspirin users defined as those using aspirin regularly during the past month. ${ }^{6}$ Defined as those using nutritional supplements during the past month. ${ }^{7}$ Coronary heart disease (CHD). 
Of the 5367 total deaths that occurred over a mean follow-up of 15 years, 1194 were attributed to cancer and 1677 were attributed to CVD. Table 2 shows the number of deaths and ASMRs of all-cause, cancer and CVD by quartile of urinary Cd concentration among US adults aged 30 years and older in NHANES 1988-1994 and 1999-2004. A greater urinary Cd concentration was positively associated with ASMRs of all-cause, cancer and CVD.

Table 2. Age-standardized mortality rates (ASMRs) of all-cause, cancer and CVD among US adults $\geq$ 30 years old in NHANES 1988-2004 by quartile of urinary Cd concentration ${ }^{1}(n=15,641)$.

\begin{tabular}{|c|c|c|c|}
\hline \multirow{2}{*}{$\begin{array}{l}\text { Urinary Cd } \\
\text { Concentration }\end{array}$} & \multirow[t]{2}{*}{ No. of Death } & \multirow{2}{*}{$\begin{array}{c}\text { Crude Mortality Rate } \\
\text { (per 100,000) }\end{array}$} & \multirow{2}{*}{$\begin{array}{c}\begin{array}{c}\text { Age-Standardized } \\
\text { Mortality Rate }^{1}\end{array} \\
\text { (per 100,000) }\end{array}$} \\
\hline & & & \\
\hline \multicolumn{4}{|c|}{ All-cause death } \\
\hline All & 5367 & 1636.5 & 2357.7 \\
\hline Quartile 1 (lowest) & 705 & 653.8 & 1918.7 \\
\hline Quartile 2 & 1062 & 1202.1 & 1915.5 \\
\hline Quartile 3 & 1534 & 2023.9 & 2332.8 \\
\hline Quartile 4 (highest) & 2066 & 3065.5 & 2935.0 \\
\hline \multicolumn{4}{|c|}{ Cancer death } \\
\hline All & 1194 & 389.2 & 486.9 \\
\hline Quartile 1 & 139 & 161.0 & 343.4 \\
\hline Quartile 2 & 198 & 225.6 & 321.6 \\
\hline Quartile 3 & 320 & 460.4 & 475.9 \\
\hline Quartile 4 & 537 & 842.8 & 733.7 \\
\hline \multicolumn{4}{|c|}{ CVD death } \\
\hline All & 1677 & 483.0 & 754.4 \\
\hline Quartile 1 & 229 & 196.2 & 680.7 \\
\hline Quartile 2 & 347 & 396.7 & 687.3 \\
\hline Quartile 3 & 501 & 611.5 & 741.2 \\
\hline Quartile 4 & 600 & 831.7 & 840.8 \\
\hline
\end{tabular}

${ }^{1}$ Age standardization is based on Census 2000 US population data.

Table 3 shows Cox proportional HRs and 95\% CIs for all-cause, cancer and CVD mortality by quartiles of urinary $\mathrm{Cd}$ concentration. In Model 1, after adjusting for age and gender, a positive linear association between urinary $\mathrm{Cd}$ and all-cause mortality was observed (HR for the highest versus the lowest quartile $2.04 ; 95 \% \mathrm{CI}=1.75$ to $2.37 ; p$-trend $<0.0001$ ). After further adjustment for potential confounders (Model 2), the association remained significant (HR for the highest versus the lowest quartile $1.38 ; 95 \% \mathrm{CI}=1.14$ to $1.68 ; p$-trend $<0.0001)$. In multivariable model 1 , adjusting for age and gender, we also observed a positive association between urinary $\mathrm{Cd}$ and cancer mortality (HR for the highest versus the lowest quartile $2.90 ; 95 \% \mathrm{CI}=2.14$ to $3.93 ; p$-trend $<0.0001$ ) and CVD mortality (HR for the highest versus the lowest quartile $1.68 ; 95 \% \mathrm{CI}=1.33$ to 2.13 ; $p$-trend $<0.0001$ ). In multivariable model 3 adjusting for cancer-related potential confounders, we observed a positive linear association between urinary $\mathrm{Cd}$ and cancer mortality (HR for the highest versus the lowest quartile $1.54 ; 95 \% \mathrm{CI}=$ 1.05 to 2.27; $p$-trend < 0.005). However, after further adjustment for CVD-related potential confounders (Model 4), the association between urinary Cd and CVD mortality was attenuated and no longer significant (HR for the highest versus the lowest quartile 1.22; $95 \% \mathrm{CI}=0.95$ to 1.57 ; p-trend 0.0502). CVD: Cardiovascular disease.

There was a marginally significant interaction between $\mathrm{Zn}$ intake and urinary $\mathrm{Cd}(p$-value $=0.08)$, so we analyzed associations within $\mathrm{Zn}$ intake subgroups. Among the lowest tertile of $\mathrm{Zn}$ consumers, there was a clear positive association between urinary $\mathrm{Cd}$ and cancer mortality (HR for Q4 vs. Q1: 1.79; 95\% CI: 1.07-3.01); however, the association was weaker among the highest $\mathrm{Zn}$ consumers (HR for 
Q4 vs. Q1: 1.66; 95\% CI: 0.80-3.41) (Table 4). There was no association between urinary Cd and CVD mortality among the lowest tertile and highest tertile of $\mathrm{Zn}$ consumers.

Table 3. Multivariable adjusted HR ( $95 \%$ CI) of all-cause, cancer and CVD mortality using Cox proportional hazards models among US adults $\geq 30$ years old in NHANES 1988-2004 by quartile of urinary Cd concentration $(n=15,641)$.

\begin{tabular}{|c|c|c|c|c|c|}
\hline & \multicolumn{4}{|c|}{ Urinary Cd Concentration ( $\mu \mathrm{g} / \mathrm{g}$ Creatinine) (Range) } & \multirow{3}{*}{$p$ for Trend } \\
\hline & Q1 $(n=3910)$ & Q2 $(n=3910)$ & Q3 $(n=3911)$ & $\mathrm{Q} 4(n=3910)$ & \\
\hline & $(0-0.27)$ & $(0.27-0.48)$ & $(0.48-0.82)$ & $(0.82-23.35)$ & \\
\hline \multicolumn{6}{|l|}{ All-cause } \\
\hline Model 11 & 1.00 & $1.13(0.96-1.33)$ & $1.44(1.26-1.64)$ & $2.04(1.75-2.37)$ & $<0.0001$ \\
\hline Model $2^{2}$ & 1.00 & $0.99(0.82-1.18)$ & $1.13(0.96-1.33)$ & $1.38(1.14-1.68)$ & $<0.0001$ \\
\hline \multicolumn{6}{|l|}{ Cancer } \\
\hline Model 1 & 1.00 & $1.01(0.75-1.36)$ & $1.68(1.31-2.14)$ & $2.90(2.14-3.93)$ & $<0.0001$ \\
\hline $\begin{array}{c}\text { Model } 3^{3} \\
\text { CVD }\end{array}$ & 1.00 & $0.84(0.60-1.18)$ & $1.21(0.90-1.62)$ & $1.54(1.05-2.27)$ & $<0.005$ \\
\hline Model 1 & 1.00 & $1.12(0.87-1.45)$ & $1.29(0.99-1.66)$ & $1.68(1.32-2.13)$ & $<0.0001$ \\
\hline Model 44 & 1.00 & $0.95(0.73-1.25)$ & $1.01(0.77-1.33)$ & $1.22(0.95-1.57)$ & 0.052 \\
\hline
\end{tabular}

${ }^{1}$ Model 1: Adjusted for age and gender. ${ }^{2}$ Model 2: Adjusted for age, gender, ethnicity, BMI, PIR, smoking status, diabetes, hypertension, alcohol consumption, saturated fatty acid intake, Zn intake, aspirin use, supplement use and history of CVD and cancer. ${ }^{3}$ Model 3: Adjusted for age, gender, ethnicity, BMI, PIR, smoking status, diabetes, alcohol consumption, $\mathrm{Zn}$ intake, aspirin use, supplement use and history of cancer. ${ }^{4}$ Model 4: Adjusted for age, gender, ethnicity, BMI, PIR, smoking status, diabetes, hypertension, alcohol consumption, saturated fatty acid intake, aspirin use, supplement use and history of CVD.

Table 4. Multivariable adjusted HR (95\% CI) of all-cause, cancer and CVD mortality using Cox proportional hazards models among US adults $\geq 30$ years old in NHANES 1988-2004 by quartile of urinary Cd concentration by tertile of $\mathrm{Zn}$ intake $(n=15,641)$.

\begin{tabular}{|c|c|c|c|c|c|}
\hline \multicolumn{6}{|c|}{ Urinary Cd Concentration ( $\mu \mathrm{g} / \mathrm{g}$ Creatinine) (Range) } \\
\hline & \multicolumn{4}{|c|}{ 1st Tertile of Dietary Zn Intake } & \multirow{3}{*}{$p$ for trend } \\
\hline & $\mathrm{Q} 1(n=1303)$ & Q2 ( $n=1304)$ & Q3 $(n=1304)$ & Q4 $(n=1303)$ & \\
\hline & $(0.00-0.31)$ & $(0.31-0.54)$ & $(0.54-0.93)$ & $(0.93-16.17)$ & \\
\hline \multicolumn{6}{|l|}{ All-cause } \\
\hline Model $1^{1}$ & 1.00 & $1.03(0.82-1.30)$ & $1.29(1.04-1.58)$ & $1.73(1.39-2.15)$ & $<0.0001$ \\
\hline Model $2^{2}$ & 1.00 & $1.00(0.80-1.25)$ & $1.04(0.81-1.34)$ & $1.25(0.96-1.63)$ & 0.062 \\
\hline \multicolumn{6}{|l|}{ Cancer } \\
\hline Model 1 & 1.00 & $1.10(0.63-1.91)$ & $2.01(1.13-3.54)$ & $3.71(2.24-6.14)$ & $<0.0001$ \\
\hline $\begin{array}{c}\text { Model } 3^{3} \\
\text { CVD }\end{array}$ & 1.00 & $0.86(0.49-1.52)$ & $1.25(0.66-2.36)$ & $1.79(1.07-3.01)$ & $<0.005$ \\
\hline Model 1 & 1.00 & $1.01(0.73-1.39)$ & $1.11(0.81-1.52)$ & $1.17(0.83-1.65)$ & 0.293 \\
\hline \multirow[t]{4}{*}{ Model 4} & 1.00 & $0.92(0.61-1.39)$ & $0.97(0.65-1.47)$ & $0.96(0.59-1.55)$ & 0.958 \\
\hline & \multicolumn{4}{|c|}{ 2nd tertile of dietary $\mathrm{Zn}$ intake } & \\
\hline & Q1 $(n=1293)$ & Q2 ( $n=1292)$ & Q3 $(n=1294)$ & Q4 (n= 1293) & \\
\hline & $(0.00-0.27)$ & $(0.27-0.47)$ & $(0.47-0.81)$ & $(0.81-21.65)$ & \\
\hline \multicolumn{6}{|l|}{ All-cause } \\
\hline Model $1^{1}$ & 1.00 & $1.15(0.91-1.46)$ & $1.33(1.08-1.64)$ & $1.94(1.55-2.44)$ & $<0.0001$ \\
\hline \multicolumn{5}{|l|}{ Cancer } & 0.067 \\
\hline Model 1 & 1.00 & $1.79(1.07-2.98)$ & $2.41(1.59-3.68)$ & $3.80(2.34-6.17)$ & $<0.0001$ \\
\hline $\begin{array}{c}\text { Model }^{3}{ }^{3} \\
\text { CVD }\end{array}$ & 1.00 & $1.47(0.83-2.60)$ & $1.45(0.96-2.21)$ & $1.79(0.95-3.37)$ & 0.070 \\
\hline Model 1 & 1.00 & $1.10(0.71-1.69)$ & $1.24(0.79-1.96)$ & $1.73(1.18-2.54)$ & $<0.001$ \\
\hline Model $4^{4}$ & 1.00 & $1.18(0.70-1.98)$ & $1.17(0.70-1.94)$ & $1.49(0.89-2.49)$ & 0.102 \\
\hline
\end{tabular}


Table 4. Cont.

\begin{tabular}{|c|c|c|c|c|c|}
\hline \multicolumn{6}{|c|}{ 3rd tertile of dietary $\mathrm{Zn}$ intake } \\
\hline & Q1 $(n=1312)$ & Q2 ( $n=1315)$ & Q3 $(n=1314)$ & Q4 $(n=1314)$ & \\
\hline & $(0-0.23)$ & $(0.23-0.42)$ & $(0.42-0.74)$ & $(0.74-23.35)$ & \\
\hline All-cause & & & & & \\
\hline Model $1^{1}$ & 1.00 & $1.09(0.83-1.43)$ & $1.62(1.22-2.14)$ & $2.38(1.89-3.04)$ & $<0.0001$ \\
\hline $\begin{array}{c}\text { Model } 22 \\
\text { Cancer }\end{array}$ & 1.00 & $0.94(0.68-1.32)$ & $1.43(1.01-2.04)$ & $1.71(1.23-2.38)$ & $<0.0001$ \\
\hline Model 1 & 1.00 & $0.71(0.38-1.33)$ & $1.29(0.75-2.23)$ & $2.22(1.30-3.77)$ & $<0.0005$ \\
\hline $\begin{array}{c}\text { Model } 3^{3} \\
\text { CVD }\end{array}$ & 1.00 & $0.65(0.29-1.42)$ & $1.23(0.61-2.47)$ & $1.66(0.80-3.41)$ & $<0.05$ \\
\hline Model 1 & 1.00 & $1.10(0.62-1.94)$ & $1.48(0.81-2.69)$ & $2.30(1.31-4.02)$ & $<0.0005$ \\
\hline Model $4^{4}$ & 1.00 & $0.75(0.41-1.35)$ & $0.93(0.51-1.67)$ & $1.32(0.77-2.26)$ & 0.081 \\
\hline
\end{tabular}

${ }^{1}$ Model 1: Adjusted for age and gender. ${ }^{2}$ Model 2: Adjusted for age, gender, ethnicity, BMI, PIR, smoking status, diabetes, hypertension, alcohol consumption, saturated fatty acid intake, $\mathrm{Zn}$ intake, aspirin use, supplement use and history of CVD and cancer. ${ }^{3}$ Model 3: Adjusted for age, gender, ethnicity, BMI, PIR, smoking status, diabetes, alcohol consumption, $\mathrm{Zn}$ intake, aspirin use, supplement use and history of cancer. ${ }^{4}$ Model 4: Adjusted for age, gender, ethnicity, BMI, PIR, smoking status, diabetes, hypertension, alcohol consumption, saturated fatty acid intake, aspirin use, supplement use and history of CVD.

Table 5 shows HRs and 95\% CIs for all-cause, cancer and CVD mortality by quartile of the Cd/Zn ratio. After adjusting for age and gender, there were positive associations between the $\mathrm{Cd} / \mathrm{Zn}$ ratio and all-cause mortality (HR for highest vs. lowest quartile (Q4 vs. Q1): 2.17; 95\% CI: 1.86-2.52; $p$-trend $<0.0001$ ), cancer mortality (HR for Q4 vs. Q1: 2.86; 95\% CI: 2.09-3.92; $p$-trend < 0.0001), and CVD mortality (HR for Q4 vs. Q1: 2.02; 95\% CI: 1.60-2.55; $p$-trend < 0.0001). After additional adjustment for related potential confounders, the significance of these positive associations between the $\mathrm{Cd} / \mathrm{Zn}$ ratio and all-cause mortality (HR for Q4 vs. Q1: 1.54; 95\% CI: 1.23-1.93; $p$-trend < 0.0005), cancer mortality (HR for Q4 vs. Q1: 1.65; 95\% CI: 1.11-2.47, $p$-trend < 0.05) and CVD mortality (HR for Q4 vs. Q1: 1.49; $95 \%$ CI: $1.18-1.88, p$-trend $<0.001)$ remained significant.

Table 5. Multivariable adjusted HR (95\% CI) of all-cause, cancer and CVD mortality using Cox proportional hazards models among US adults $\geq 30$ years old in NHANES 1988-2004 by quartile of $\mathrm{Cd} / \mathrm{Zn}$ ratio $(n=15,641)$.

\begin{tabular}{|c|c|c|c|c|c|}
\hline & \multicolumn{4}{|c|}{ 1988-2004 } & \multirow{4}{*}{$p$ for Trend } \\
\hline & \multicolumn{4}{|c|}{ Cd/Zn Ratio (Range) } & \\
\hline & Q1 $(n=3910)$ & $\mathrm{Q} 2(n=3910)$ & Q3 $(n=3911)$ & $\mathrm{Q} 4(n=3910)$ & \\
\hline & $(0-0.002)$ & $(0.002-0.005)$ & $(0.005-0.011)$ & $(0.011-6.464)$ & \\
\hline \multicolumn{6}{|l|}{ All-cause } \\
\hline Model $1^{1}$ & 1.00 & $1.36(1.16-1.60)$ & $1.68(1.43-1.96)$ & $2.17(1.86-2.52)$ & $<0.0001$ \\
\hline Model $2^{2}$ & 1.00 & $1.27(1.04-1.57)$ & $1.29(1.05-1.59)$ & $1.54(1.23-1.93)$ & $<0.0005$ \\
\hline \multicolumn{6}{|l|}{ Cancer } \\
\hline Model 1 & 1.00 & $1.43(1.06-1.92)$ & $1.87(1.36-2.59)$ & $2.86(2.09-3.92)$ & $<0.0001$ \\
\hline Model $3^{3}$ & 1.00 & $1.27(0.91-1.76)$ & $1.33(0.90-1.96)$ & $1.65(1.11-2.47)$ & $<0.05$ \\
\hline \multicolumn{6}{|l|}{ CVD } \\
\hline Model 1 & 1.00 & $1.24(0.95-1.62)$ & $1.71(1.30-2.26)$ & $2.02(1.60-2.55)$ & $<0.0001$ \\
\hline Model $4^{4}$ & 1.00 & $1.17(0.86-1.58)$ & $1.28(0.99-1.67)$ & $1.49(1.18-1.88)$ & $<0.001$ \\
\hline
\end{tabular}

${ }^{1}$ Model 1: Adjusted for age and gender. ${ }^{2}$ Model 2: Adjusted for age, gender, ethnicity, BMI, PIR, smoking status, diabetes, hypertension, alcohol consumption, saturated fatty acid intake, aspirin use, supplement use and history of CVD and cancer. ${ }^{3}$ Model 3: Adjusted for age, gender, ethnicity, BMI, PIR, smoking status, diabetes, alcohol consumption, aspirin use, supplement use and history of cancer. ${ }^{4}$ Model 4: Adjusted for age, gender, ethnicity, BMI, PIR, smoking status, diabetes, hypertension, alcohol consumption, saturated fatty acid intake, aspirin use, supplement use and history of CVD. 


\section{Discussion}

Using nationally representative data, US adults with the highest urinary $\mathrm{Cd}$ tended to be older, female, current heavy smokers, normal weight status and lower levels of income, which is consistent with previous findings based on NHANES 1999-2012 data [42]. Moreover, our findings are in agreement with studies showing that urinary $\mathrm{Cd}$ was negatively associated with BMI in NHANES 1999-2002 [15,43]. The current study also found that higher urinary Cd was associated with CVD risk factors such as hypertension, aspirin use, and history of CHD or stroke, consistently with a previous report linking urinary $\mathrm{Cd}$ to CVD [44]. We also found that greater urinary Cd was associated with cancer history, which is consistent with previous studies showing that higher urinary Cd level was associated with total cancer, lung cancer [45] and breast cancer risk in Japanese women [46].

This study found that greater urinary $\mathrm{Cd}$ is associated with greater all-cause mortality and cancer mortality after adjusting for important potential confounders. These results are consistent with previous studies reporting that urinary $\mathrm{Cd}$ levels were associated with a greater risk of all-cause cancer mortality $[12,15,47]$. Garcia-Esquinas [12] et al. showed that Cd exposure was positively associated with total cancer mortality and with mortality from cancers of the lung and pancreas. While Menke [15] and Larsson [47] reported that urinary Cd was positively associated with CVD mortality, the current study shows that the association between urinary Cd and CVD mortality was diminished after adjusting for all relevant covariates and was marginally significant (HR for the highest versus the lowest quartile $1.22 ; 95 \% \mathrm{CI}=0.95$ to $1.57 ; p$-trend 0.0502 ).

Several studies reported that $\mathrm{Zn}$ can reduce the risk of $\mathrm{Cd}$ toxicity by competitive interactions between $\mathrm{Zn}$ and $\mathrm{Cd}[9,16]$. Our group previously reported that $\mathrm{Zn}$ intake is associated with lower $\mathrm{Cd}$ burden [21] and with lower urinary $\mathrm{Cd}$ among non-smokers [48]. In our fully adjusted analysis, there was a marginally significant interaction between urinary $\mathrm{Cd}$ and $\mathrm{Zn}$ intake ( $p$-value $=0.08$ ). This finding suggests that the relationship between $\mathrm{Zn}$ intake and urinary $\mathrm{Cd}$ may differ by $\mathrm{Zn}$ intake level, and that both $\mathrm{Zn}$ intake and $\mathrm{Cd}$ exposure may need to be considered together to precisely predict mortality risk. We therefore investigated the associations of urinary $\mathrm{Cd}$ with mortality by $\mathrm{Zn}$ intake level. We found that greater urinary $\mathrm{Cd}$ was associated with greater cancer mortality only among the lowest tertile of $\mathrm{Zn}$ consumers. Because higher $\mathrm{Zn}$ intakes appeared to reduce the strength of the association between $\mathrm{Cd}$ and mortality, we investigated the combined relationship of $\mathrm{Cd}$ exposure and $\mathrm{Zn}$ intake with mortality using the $\mathrm{Cd} / \mathrm{Zn}$ ratio. As the association of urinary $\mathrm{Cd}$ with mortality might be modified by dietary $\mathrm{Zn}$ level, the $\mathrm{Cd} / \mathrm{Zn}$ ratio may be a helpful metric for investigating the combined relationship of $\mathrm{Cd}$ exposure, $\mathrm{Zn}$ intake and mortality. We found a clear positive association between the $\mathrm{Cd} / \mathrm{Zn}$ ratio cancer mortality, consistent with a previous study by Lin et al. showing a positive association between $\mathrm{Cd}$ exposure and cancer mortality among older adults in NHANES III with low $\mathrm{Zn}$ intake levels [23]. Lin et al. [23] also showed that the $\mathrm{Zn}$-to-Cd ratio was more strongly associated with cancer mortality risk than either $\mathrm{Zn}$ intake or urinary $\mathrm{Cd}$ alone. In addition to confirming the association between the $\mathrm{Cd} / \mathrm{Zn}$ ratio and cancer mortality, the current study also demonstrated that the $\mathrm{Cd} / \mathrm{Zn}$ ratio is an important predictor of all-cause and CVD mortality. Furthermore, we characterized these associations, showing that linear trends existed between the $\mathrm{Cd} / \mathrm{Zn}$ ratio and all-cause, cancer and CVD mortality in adjusted models.

This study is strengthened by its use of a large, nationally representative sample of the US adult population. This large and diverse sample allows for the examination of the relationships between urinary $\mathrm{Cd}$, mortality, and $\mathrm{Zn}$ intake across participants with varying lifestyle and sociodemographic characteristics. Additionally, this study included a large number of deaths and a long-term follow-up of 15 years. However, this study has several limitations. Firstly, estimation of $\mathrm{Zn}$ intake was based on one day of dietary recall data, which may not accurately reflect usual intake of individual participants. Secondly, competing risks can be present between CVD and cancer mortality. Thirdly, even though we tried to match variables between datasets of 1988-1994 and those of 1999-2004, there might be systematic error in combining two datasets. Finally, residual confounding factors might be present in this analysis, although we attempted to adjust for all relevant covariates available in the NHANES dataset. 


\section{Conclusions}

In conclusion, a greater $\mathrm{Cd} / \mathrm{Zn}$ ratio was associated with greater mortality from all causes, cancer, and CVD in fully adjusted models, indicating that the $\mathrm{Cd} / \mathrm{Zn}$ ratio, rather than urinary $\mathrm{Cd}$ or $\mathrm{Zn}$ intake alone, may be an important predictor of mortality from all causes, cancer, and CVD. Future research should be conducted to establish recommendation for optimal $\mathrm{Zn}$ intake for protecting against $\mathrm{Cd}$ toxicity and to explain the relationship between $\mathrm{Cd}$-related disease risk and mortality using biomarkers in a prospective cohort study.

Author Contributions: Conceptualization, K.K. and O.K.C.; Methodology, K.K. and M.M.M.; Analysis, K.K.; Writing-Original Draft Preparation, K.K.; Writing-Review \& Editing, K.K., M.M.M., J.R.S., K.H., D.M., H.N. and O.K.C.; Supervision, O.K.C. All authors have read and agreed to the published version of the manuscript.

Funding: This research received no external funding.

Acknowledgments: Where authors are identified as personnel of the International Agency for Research on Cancer/World Health Organization, the authors alone are responsible for the views expressed in this article and they do not necessarily represent the decisions, policy or views of the International Agency for Research on Cancer/World Health Organization.

Conflicts of Interest: The authors declare no conflict of interest.

\section{References}

1. Agency for Toxic Substances and Disease Registry (2012) Toxicological Profile for Cadmium. Available online: https://www.atsdr.cdc.gov/ToxProfiles/tp5.pdf (accessed on 5 November 2019).

2. Pappas, R.S.; Polzin, G.M.; Zhang, L.; Watson, C.H.; Paschal, D.C.; Ashley, D.L. Cadmium, lead, and thallium in mainstream tobacco smoke particulate. Food Chem. Toxicol. 2006, 44, 714-723. [CrossRef] [PubMed]

3. Mortensen, M.E.; Wong, L.Y.; Osterloh, J.D. Smoking status and urine cadmium above levels associated with subclinical renal effects in U.S. adults without chronic kidney disease. Int. J. Hyg. Environ. Health 2011, 214, 305-310. [CrossRef] [PubMed]

4. Godt, J.; Scheidig, F.; Grosse-Siestrup, C.; Esche, V.; Brandenburg, P.; Reich, A.; Groneberg, D.A. The toxicity of cadmium and resulting hazards for human health. J. Occup. Med. Toxicol. 2006, 1, 22. [CrossRef] [PubMed]

5. Peters, J.L.; Perlstein, T.S.; Perry, M.J.; McNeely, E.; Weuve, J. Cadmium exposure in association with history of stroke and heart failure. Environ. Res. 2010, 110, 199-206. [CrossRef] [PubMed]

6. Bernhoft, R.A. Cadmium toxicity and treatment. Sci. World J. 2013, 2013, 394652. [CrossRef] [PubMed]

7. Gallagher, C.M.; Kovach, J.S.; Meliker, J.R. Urinary cadmium and osteoporosis in U.S. Women $\geq 50$ years of age: NHANES 1988-1994 and 1999-2004. Environ. Health Perspect. 2008, 116, 1338-1343. [CrossRef] [PubMed]

8. Hartwig, A. Cadmium and cancer. Met. Ions Life Sci. 2013, 11, 491-507.

9. McCarty, M.F. Zinc and multi-mineral supplementation should mitigate the pathogenic impact of cadmium exposure. Med. Hypotheses 2012, 79, 642-648. [CrossRef]

10. Li, H.; Fagerberg, B.; Sallsten, G.; Borné, Y.; Hedblad, B.; Engström, G.; Barregard, L.; Andersson, E.M. Smoking-induced risk of future cardiovascular disease is partly mediated by cadmium in tobacco: Malmo Diet and Cancer Cohort Study. Environ. Health 2019, 18, 56. [CrossRef]

11. Deering, K.E.; Callan, A.C.; Prince, R.L.; Lim, W.H.; Thompson, P.L.; Lewis, J.R.; Hinwood, A.L.; Devine, A. Low-level cadmium exposure and cardiovascular outcomes in elderly Australian women: A cohort study. Int. J. Hyg. Environ. Health 2018, 221, 347-354. [CrossRef]

12. Garcia-Esquinas, E.; Pollan, M.; Tellez-Plaza, M.; Francesconi, K.A.; Goessler, W.; Guallar, E.; Umans, J.G.; Yeh, J.; Best, L.G.; Navas-Acien, A. Cadmium exposure and cancer mortality in a prospective cohort: The strong heart study. Environ. Health Perspect. 2014, 122, 363-370. [CrossRef] [PubMed]

13. Suwazono, Y.; Nogawa, K.; Morikawa, Y.; Nishijo, M.; Kobayashi, E.; Kido, T.; Nakagawa, H.; Nogawa, K. All-cause mortality increased by environmental cadmium exposure in the Japanese general population in cadmium non-polluted areas. J. Appl. Toxicol. 2015, 35, 817-823. [CrossRef] [PubMed]

14. Nawrot, T.S.; Van Hecke, E.; Thijs, L.; Richart, T.; Kuznetsova, T.; Jin, Y.; Vangronsveld, J.; Roels, H.A.; Staessen, J.A. Cadmium-related mortality and long-term secular trends in the cadmium body burden of an environmentally exposed population. Environ. Health Perspect. 2008, 116, 1620-1628. [CrossRef] [PubMed] 
15. Menke, A.; Muntner, P.; Silbergeld, E.K.; Platz, E.A.; Guallar, E. Cadmium levels in urine and mortality among U.S. adults. Environ. Health Perspect. 2009, 117, 190-196. [CrossRef] [PubMed]

16. Brzoska, M.M.; Moniuszko-Jakoniuk, J. Interactions between cadmium and zinc in the organism. Food Chem. Toxicol. 2001, 39, 967-980. [CrossRef]

17. Roels, H.A.; Hoet, P.; Lison, D. Usefulness of biomarkers of exposure to inorganic mercury, lead, or cadmium in controlling occupational and environmental risks of nephrotoxicity. Ren. Fail. 1999, 21, 251-262. [CrossRef]

18. Brzoska, M.M.; Roszczenko, A.; Galazyn-Sidorczuk, M.; Majewska, K. Zinc supplementation can protect from enhanced risk of femoral neck fracture in male rats chronically exposed to cadmium. Exp. Toxicol. Pathol. 2011, 63, 491-498. [CrossRef]

19. Rogalska, J.; Pilat-Marcinkiewicz, B.; Brzoska, M.M. Protective effect of zinc against cadmium hepatotoxicity depends on this bioelement intake and level of cadmium exposure: A study in a rat model. Chem. Biol. Interact. 2011, 193, 191-203. [CrossRef]

20. Bulat, Z.P.; Djukic-Cosic, D.; Malicevic, Z.; Bulat, P.; Matović, V. Zinc or magnesium supplementation modulates cd intoxication in blood, kidney, spleen, and bone of rabbits. Biol. Trace Elem. Res. 2008, 124, 110-117. [CrossRef]

21. Vance, T.M.; Chun, O.K. Zinc Intake Is Associated with Lower Cadmium Burden in U.S. Adults. J. Nutr. 2015, 145, 2741-2748. [CrossRef]

22. Van Wijngaarden, E.; Singer, E.A.; Palapattu, G.S. Prostate-specific antigen levels in relation to cadmium exposure and zinc intake: Results from the 2001-2002 National Health and Nutrition Examination Survey. Prostate 2008, 68, 122-128. [CrossRef] [PubMed]

23. Lin, Y.S.; Caffrey, J.L.; Lin, J.W.; Bayliss, D.; Faramawi, M.F.; Bateson, T.F.; Sonawane, B. Increased risk of cancer mortality associated with cadmium exposures in older Americans with low zinc intake. J. Toxicol. Environ. Health A 2013, 76, 1-15. [CrossRef] [PubMed]

24. U.S. Department of Health and Human Services. National Health and Nutrition Examination Survey III, 1988-1994. Centers for Disease Control and Prevention (CDC). Available online: https://wwwn.cdc.gov/ nchs/nhanes/nhanes3/DataFiles.aspx (accessed on 5 November 2019).

25. U.S. Department of Health and Human Services. National Health and Nutrition Examination Survey Data 1999-2000. Centers for Disease Control and Prevention (CDC). Available online: https://wwwn.cdc.gov/ nchs/nhanes/continuousnhanes/default.aspx?Begin Year=1999 (accessed on 5 November 2019).

26. U.S. Department of Health and Human Services. National Health and Nutrition Examination Survey Data 2001-2002. Centers for Disease Control and Prevention (CDC). Available online: https://wwwn.cdc.gov/ nchs/nhanes/continuousnhanes/default.aspx?Begin Year=2001 (accessed on 5 November 2019).

27. U.S. Department of Health and Human Services. National Health and Nutrition Examination Survey Data 2003-2004. Centers for Disease Control and Prevention (CDC). Available online: https://wwwn.cdc.gov/ nchs/nhanes/continuousnhanes/default.aspx?Begin Year=2003 (accessed on 5 November 2019).

28. U.S. Cancer Statistics Working Group. U.S. Cancer Statistics Data Visualizations Tool, Based on November 2018 Submission Data (1999-2016); June 2019, U.S.; Department of Health and Human Services, Centers for Disease Control and Prevention and National Cancer Institute: Atlanta, GA, USA, 2019.

29. Kenneth, D.K.; Sherry, L.; Murphy, J.X.; Elizabeth, A. National Vital Statistics Reports; Department of Health and Human Services; Department of Health and Human Services, Centers for Disease Control and Prevention, National Center for Health Statistics: Hyattsville, MD, USA, 2019.

30. Gunter, E.W.; Lewis, B.G.; Koncikowski, S.M. Laboratory Procedures Used for the Third National Health and Nutrition Examination Survey (NHANES III), 1988-1994; Atlanta, G.A., Ed.; Department of Health and Human Services, Centers for Disease Control and Prevention: Cincinnati, OH, USA, 1996.

31. National Center for Health Statistics, NHANES. Laboratory/Medical Technologists Procedures Manual (LPM); Centers for Disease Control and Prevention: Hyattsville, MD, USA, 2004.

32. Jarup, L. Cadmium overload and toxicity. Nephrol. Dial. Transplant. 2002, 17, 35-39. [CrossRef] [PubMed]

33. National Center for Health Statistics. Office of Analysis and Epidemiology, NCHS 2011 Linked Mortality Files Matching Methodology, September, 2013; National Center for Health Statistics: Hyattsville, MD, USA, 2013; Available online: http://www.cdc.gov/nchs/data_access/data_linkage/mortality/linkage_methods_analytical_ support/2011_linked_mortality_file_matching_methodology.pdf (accessed on 5 November 2019).

34. World Health Organization (WHO). International Statistical Classification of Disease and Related Health Problems, Tenth Revision (ICD-10); WHO: Geneva, Switzerland, 1992. 
35. Ronksley, P.E.; Ronksley, P.E.; Brien, S.E.; Turner, B.J.; Mukamal, K.J.; Ghali, W.A. Association of alcohol consumption with selected cardiovascular disease outcomes: A systematic review and meta-analysis. BMJ 2011, 342, d671. [CrossRef] [PubMed]

36. Xi, B.; Veeranki, S.P.; Zhao, M.; Ma, C.; Yan, Y.; Mi, J. Relationship of Alcohol Consumption to All-Cause, Cardiovascular, and Cancer-Related Mortality in U.S. Adults. J. Am. Coll. Cardiol. 2017, 70, 913-922. [CrossRef] [PubMed]

37. Coughlin, S.S.; Calle, E.E.; Patel, A.V.; Thun, M.J. Predictors of pancreatic cancer mortality among a large cohort of United States adults. Cancer Causes Control 2000, 11, 915-923. [CrossRef] [PubMed]

38. Ratnasinghe, L.D.; Graubard, B.I.; Kahle, L.; Tangrea, J.A.; Taylor, P.R.; Hawk, E. Aspirin use and mortality from cancer in a prospective cohort study. Anticancer Res. 2004, 24, 3177-3184.

39. Aluli, N.E.; Reyes, P.W.; Brady, S.K.; Tsark, J.U.; Jones, K.L.; Mau, M.; Howard, W.J.; Howard, B.V. All-cause and CVD mortality in Native Hawaiians. Diabetes Res. Clin. Pract. 2010, 89, 65-71. [CrossRef]

40. Dacey, L.J.; Munoz, J.J.; Johnson, E.R.; Leavitt, B.J.; Maloney, C.T.; Morton, J.R.; Olmstead, E.M.; Birkmeyer, J.D.; O'Connor, G.T. Effect of preoperative aspirin use on mortality in coronary artery bypass grafting patients. Ann. Thorac. Surg. 2000, 70, 1986-1990. [CrossRef]

41. Lantz, P.M.; House, J.S.; Lepkowski, J.M.; Williams, D.R.; Mero, R.P.; Chen, J. Socioeconomic factors, health behaviors, and mortality: Results from a nationally representative prospective study of US adults. JAMA 1998, 279, 1703-1708. [CrossRef]

42. Jain, R.B. Factors affecting the variability in the observed levels of cadmium in blood and urine among former and current smokers aged 20-64 and $\geq 65 y e a r s$. Environ. Sci. Pollut. Res. Int. 2017, 24, 8837-8851. [CrossRef] [PubMed]

43. Padilla, M.A.; Elobeid, M.; Ruden, D.M.; Allison, D.B. An examination of the association of selected toxic metals with total and central obesity indices: NHANES 99-02. Int. J. Environ. Res. Public Health 2010, 7, 3332-3347. [CrossRef] [PubMed]

44. Hecht, E.M.; Arheart, K.L.; Lee, D.J.; Hennekens, C.H.; Hlaing, W.M. Interrelation of Cadmium, Smoking, and Cardiovascular Disease (from the National Health and Nutrition Examination Survey). Am. J. Cardiol. 2016, 118, 204-209. [CrossRef] [PubMed]

45. Nawrot, T.S.; Martens, D.S.; Hara, A.; Plusquin, M.; Vangronsveld, J.; Roels, H.A.; Staessen, J.A. Association of total cancer and lung cancer with environmental exposure to cadmium: The meta-analytical evidence. Cancer Causes Control 2015, 26, 1281-1288. [CrossRef] [PubMed]

46. Nagata, C.; Nagao, Y.; Nakamura, K.; Wada, K.; Tamai, Y.; Tsuji, M.; Yamamoto, S.; Kashiki, Y. Cadmium exposure and the risk of breast cancer in Japanese women. Breast Cancer Res. Treat. 2013, 138, 235-239. [CrossRef] [PubMed]

47. Larsson, S.C.; Wolk, A. Urinary cadmium and mortality from all causes, cancer and cardiovascular disease in the general population: Systematic review and meta-analysis of cohort studies. Int. J. Epidemiol. 2016, 45, 782-791. [CrossRef]

48. Kim, K.; Melough, M.M.; Vance, T.M.; Kim, D.; Noh, H.; Koo, S.I.; Chun, O.K. The relationship between zinc intake and cadmium burden is influenced by smoking status. Food Chem. Toxicol. 2019, 125, 210-216. [CrossRef]

(C) 2019 by the authors. Licensee MDPI, Basel, Switzerland. This article is an open access article distributed under the terms and conditions of the Creative Commons Attribution (CC BY) license (http://creativecommons.org/licenses/by/4.0/). 